\title{
Risk factors for and outcomes of postoperative complications in adult patients with moyamoya disease
}

\author{
Meng Zhao, MD,-3 Xiaofeng Deng, MD, ${ }^{1-3}$ Dong Zhang, MD, ${ }^{1-3}$ Shuo Wang, MD, ${ }^{1-3}$ Yan Zhang, MD, ${ }^{1-3}$ \\ Rong Wang, MD, ${ }^{1-3}$ and Jizong Zhao, MD ${ }^{1-3}$
}

${ }^{1}$ Department of Neurosurgery, Beijing Tiantan Hospital, Capital Medical University; ${ }^{2}$ China National Clinical Research Center for Neurological Diseases; and ${ }^{3}$ Center of Stroke, Beijing Institute for Brain Disorders, Beijing, People's Republic of China

OBJECTIVE The risk factors and clinical significance of postoperative complications in moyamoya disease are still unclear. The aim of this study was to investigate the predictors of postoperative complications in moyamoya disease and examine the impact of complications on outcomes.

METHODS The authors reviewed consecutive cases involving adult moyamoya disease patients who underwent indirect, direct, or combined bypass surgery in their hospital between 2009 and 2015. Preoperative clinical characteristics and radiographic features were recorded. Postoperative complications within 14 days after surgery were examined. Multivariate logistic regression analyses were performed to identify the risk factors for either postoperative ischemia or postoperative cerebral hyperperfusion. Outcome data, including recurrent strokes and neurological status (modified Rankin Scale [mRS]) during follow-up, were collected. Outcomes were compared between patients who had complications with those without complications, using propensity-score analysis to account for between-group differences in baseline characteristics.

RESULTS A total of 500 patients (610 hemispheres) were included in this study. Postoperative complications were observed in 74 operations (12.1\%), including new postoperative ischemia in 30 cases (4.9\%), hyperperfusion in 27 (4.4\%), impaired wound healing in $12(2.0 \%)$, and subdural effusion in $6(1.0 \%)$. The complication rates for different surgery types were as follows: $12.6 \%(n=25)$ for indirect bypass, $12.7 \%(n=37)$ for direct bypass, and $10.0 \%(n=12)$ for combined bypass $(p=0.726)$. Postoperative ischemic complications occurred in 30 hemispheres $(4.9 \%)$ in 30 different patients, and postoperative symptomatic hyperperfusion occurred after 27 procedures (4.4\%). Advanced Suzuki stage (OR 1.669, 95\% $\mathrm{Cl} 1.059-2.632, p=0.027$ ) and preoperative ischemic presentation (OR 5.845, 95\% Cl 1.654-20.653, $p=0.006)$ were significantly associated with postoperative ischemia. Preoperative ischemic presentation (OR 5.73, 95\% Cl 1.27-25.88, $p=0.023$ ) and admission modified Rankin Scale (mRS) score (OR 1.81, 95\% Cl 1.06-3.10, $p=0.031$ ) were significantly associated with symptomatic postoperative cerebral hyperperfusion syndrome (CHS). Compared with patients without postoperative complications, patients who experienced any postoperative complications had longer hospital stays and worse mRS scores at discharge (both $p<0.0001$ ). At the final follow-up, no significant differences in functional disability (mRS score 3-6, $11.9 \%$ vs 4.5\%, $p=0.116)$ and future stroke events $(p=0.513)$ between the 2 groups were detected.

CONCLUSIONS Advanced Suzuki stage and preoperative ischemic presentation were independent risk factors for postoperative ischemia; the $\mathrm{mRS}$ score on admission and preoperative ischemic presentation were independently associated with postoperative CHS. Although patients with postoperative complications had worse neurological status at discharge, postoperative complications had no associations with future stroke events or functional disability during follow-up.

https://thejns.org/doi/abs/10.3171/2017.10.JNS171749

KEYWORDS moyamoya disease; complication; revascularization; stroke; outcome; adult; vascular disorders

ABBREVIATIONS CHS = cerebral hyperperfusion syndrome; DSA = digital subtraction angiography; $E D A S=$ encephaloduroarteriosynangiosis; ICA = internal carotid artery; $\mathrm{MCA}$ = middle cerebral artery; MMD = moyamoya disease; MRA = MR angiography; $m R S$ = modified Rankin Scale; PCA = posterior cerebral artery; $S T A=$ superficial temporal artery; $\mathrm{TIA}=$ transient ischemic attack.

SUBMITTED July 18, 2017. ACCEPTED October 18, 2017.

INCLUDE WHEN CITING Published online March 30, 2018; DOI: 10.3171/2017.10.JNS171749. 
$\mathrm{M}$ OyAmoya disease (MMD) is characterized by chronic, progressive stenosis or occlusion of unknown cause that affects the distal internal carotid artery (ICA) or the proximal middle cerebral artery (MCA), along with development of collateral vessels at the base of the brain. ${ }^{40}$ Although there has been no randomized controlled trial comparing surgical or medical treatment in MMD patients, revascularization surgery has generally been accepted as an effective measure for treatment of MMD in the long term. ${ }^{23,34,38}$ However, the incidence of postoperative complications such as cerebral ischemia has been increasingly reported, with these complications being more frequent in adult MMD patients than in pediatric patients. ${ }^{21}$ Also, patients with MMD have a significantly higher risk for cerebral hyperperfusion syndrome (CHS) than those with other occlusive cerebrovascular diseases. ${ }^{10,13}$ These procedure-related complications could lead to potential neurological deterioration and permanent neurological deficits. ${ }^{1,2,26}$ Accordingly, preventing and managing these immediate postoperative complications are essential for ensuring the benefits of surgical treatment. ${ }^{27}$ Reports on postoperative complications in MMD patients after revascularization are scant. The risk factors of postoperative complications are still unclear. The impact of those complications on outcomes after revascularization remains obscure. Thus, we conducted this study to explore the risk factors associated with postoperative complications and to clarify the relation of these complications with surgical outcomes.

\section{Methods}

\section{Study Design and Participants}

The participants included in this study were from a single-center cohort of MMD patients treated between 2009 and 2015. ${ }^{4}$ Consecutive cases involving MMD patients who underwent surgical revascularization at the Stroke Center of Beijing Tiantan Hospital were reviewed. The diagnosis of MMD was confirmed with digital subtraction angiography (DSA) and/or MR angiography (MRA) based on the criteria of the Research Committee on Spontaneous Occlusion of the Circle of Willis (2012) ${ }^{36}$ Patients with moyamoya syndrome due to other clear disease entities were excluded from the current study. Pediatric patients (less than 18 years old) were also excluded. Written informed consent was obtained from all patients. The study was performed according to the guidelines of the Helsinki Declaration and was approved by the ethics committee of Beijing Tiantan Hospital.

\section{Perioperative Evaluations}

We reviewed the medical records of the included patients to assess possible risk factors associated with the development of postoperative complications, such as age, sex, onset manifestation, past medical history, neurological status, and imaging findings. We categorized the onset manifestation into 3 main types: ischemic (cerebral infarction and transient ischemic attack [TIA]), hemorrhagic, and nonspecific symptoms (headache, epilepsy, and asymptomatic). A standardized perioperative management protocol was applied in all cases, and all patients under- went a CT scan on postoperative day 1. Repeat CT or MRI scans were performed in cases of postoperative neurological deterioration. Surgical complications, including new infarction, hyperperfusion syndrome, impaired wound healing, and subdural effusion were recorded. Clinically evident neurological deficits that occurred within 14 days after surgery and could be explained by cerebral hypoperfusion were categorized as postoperative ischemia, which was confirmed as new signs of ischemia on postoperative imaging. CHS was diagnosed if the patient demonstrated a focal seizure, or reversible deterioration of consciousness level with behavioral and/or speech abnormalities, or intracranial hemorrhage on $\mathrm{CT}$, along with absence of a definite new infarction on a brain CT scan and/or diffusion-weighted MRI. ${ }^{43}$ Neurological status was evaluated with the modified Rankin Scale (mRS) on admission, at discharge, and at follow-up. ${ }^{29}$ Patients were followed up by means of clinic or telephone interviews at 3-6 months after surgery and annually thereafter. Doctors who performed follow-up assessments were blinded to surgical groups.

\section{Surgical Modalities}

The principles of the surgical strategies were as follows. First, the indication for revascularization was based on the guidelines set by the Japanese Ministry of Health and Welfare. ${ }^{36}$ The symptomatic and hemodynamically affected hemisphere was the preferred side for revascularization surgery. Second, if the patient's symptoms were significantly improved after the first surgery and the patient did not have symptoms that could be ascribed to the contralateral hemisphere, operative treatment of the contralateral hemisphere was not considered. Otherwise, surgery on the contralateral hemisphere was performed.

The surgical procedures of MMD could be divided into 3 categories: indirect bypass, direct bypass, and combined bypass. Direct bypass involves the end-to-side anastomosis of the branches of the superficial temporal artery (STA) to the cortical branches of middle cerebral artery (MCA). The graft patency was routinely confirmed with intraoperative indocyanine green videoangiography or postoperative CT angiography. Indirect bypass included encephaloduroarteriosynangiosis (EDAS) and multiple burr holes. For EDAS, the STA and accompanying cuff of galea connective tissue were exposed. The STA was sutured onto the brain surface after being dissected free. For multiple burr holes, $5-15$ burr holes were placed over the hypoperfusion brain area; the underlying dura was incised and separated. If both direct and indirect bypass procedures were performed on a single hemisphere, the operation was classified as a combined bypass procedure.

\section{Follow-Up}

Patients were followed up by face-to-face or telephone interviews at 3-6 months after surgery and annually thereafter. Doctors performing follow-up assessments were blinded to baseline information. Clinical outcomes including recurrent strokes (intracranial hemorrhage and cerebral ischemia) and neurological status (mRS) were collected during follow-ups. 


\section{Statistical Analysis and Propensity-Score Matching}

The categorical variables are presented as counts (with percentages); continuous variables are presented as the means \pm standard deviations. The Pearson chi-square test, Fisher exact test, or Mann-Whitney U-test were used to compare categorical variables as appropriate. Two-tailed t-tests were used to compare continuous variables. Odds ratios (ORs) and 95\% confidence intervals (CIs) for postoperative ischemia or hyperperfusion for potential risk factors were calculated by univariate and multivariate logistic regression analyses.

We used propensity-score analysis methodology to compare outcomes of patients in the complications group with those of patients in the no-complications group. This approach was used to reduce the imbalance in baseline patient characteristics between the 2 groups. On the basis of covariates from the logistic model, we generated a propensity score for each patient with respect to age, sex, presentation type, hypertension, diabetes, admission $\mathrm{mRS}$ score, Suzuki stage, posterior cerebral artery (PCA) involvement, and surgery type. We used the nearest matching algorithm with a 1:1 ratio. The outcomes of interest were stroke events and mRS scores during follow-up. Kaplan-Meier survival analysis was used to compare the stroke-free survival rates between patients with and without complications. A p value $<0.05$ was considered to be statistically significant. Statistical analysis was performed using the R statistical program (version 3.3.1; R core team).

\section{Results}

\section{Baseline Characteristics and Incidence of Postoperative Complications}

A total of 500 patients (227 males and 273 females) were included in the study. Among them, 110 patients (40 males and 70 females) received a second operation on the contralateral side, for a total of 610 procedures. The patients' mean age at the time of these procedures was 37.5 \pm 9.4 years. Analyzed per procedure, the clinical presentation was ischemia in 371 (60.8\%) cases, hemorrhage in 196 (32.1\%), and nonspecific symptoms in 43 (7.0\%).

Operative procedures included 199 indirect bypasses, 291 direct bypasses, and 120 combined bypasses. Postoperative complications were observed after $74(12.1 \%)$ of the operations, including new postoperative ischemia in 30 cases (4.9\%), hyperperfusion in 27 (4.4\%), impaired wound healing in $12(2.0 \%)$, and subdural effusion in 6 $(1.0 \%)$. One patient had intracerebral hemorrhage after postoperative ischemia. Stratified by surgery type, the numbers of complications and rates were as follows: 25 (12.6\%) for indirect bypass, 37 (12.7\%) for direct bypass, and $12(10.0 \%)$ for combined bypass $(\mathrm{p}=0.726)$. A comparison of the baseline characteristics of patients with any postoperative complications and those without postoperative complications is presented in Table 1. Patients with postoperative complications tended to be male, be older, have higher Suzuki stages of MMD, and have a higher prevalence of diabetes (all $\mathrm{p}<0.05$ ).

\section{Postoperative Ischemia}

Postoperative ischemic complications occurred in 30 hemispheres (4.9\%) in 30 patients. In 11 cases, the complications occurred after indirect bypass; in 12, after direct bypass; and in 7, after combined bypass. Thirteen patients with ischemic complications had neurological sequelae and had worse neurological status at discharge (higher $\mathrm{mRS}$ score) than at admission.

The prevalence of preoperative ischemic presentation (90.0\% vs 59.3\%, $\mathrm{p}=0.001)$ and distribution of Suzuki stages $(p=0.005)$ differed significantly between the group with new-onset postoperative ischemia $(\mathrm{n}=30)$ and the group that did not have postoperative ischemic complications $(\mathrm{n}=580)$ (Table 2). Univariate analysis showed that preoperative ischemic presentation and Suzuki stage were significantly associated with postoperative ischemia. After adjustment for cofounding variables in multivariate analysis, preoperative ischemic presentation (OR 5.845, 95\% CI 1.654-20.653, $\mathrm{p}=0.006)$ and Suzuki stage (OR $1.669,95 \%$ CI $1.059-2.632, \mathrm{p}=0.027)$ remained significantly associated with postoperative ischemia (Table 3 ).

\section{Postoperative Hyperperfusion Complications}

Symptomatic hyperperfusion occurred after 27 (4.4\%) procedures. Eight of these complications occurred after indirect bypass, 16 occurred after direct bypass, and 3 occurred after combined bypass (Table 2), resulting in rates of $4.0 \%, 5.5 \%$, and $2.5 \%$, respectively, for the 3 types of surgery. The symptoms of CHS included cerebral hemorrhage in 8 patients, epilepsy in 7, aphasia/dysarthria in 10 , and limb weakness in 2 . The timing of onset of the patients' neurological deficit symptoms varied from 1 to 6 days (mean $3.3 \pm 1.8$ days) after surgery and most of these symptoms disappeared completely after 1-12 days (mean $4.0 \pm 3.1$ days). The mRS score was worse at discharge than at admission in $6(22 \%)$ of the 27 patients who experienced symptomatic postoperative hyperperfusion, whereas the remaining 21 patients recovered to their preoperative state or better.

Patients with postoperative hyperperfusion were more likely to be male and they had a higher prevalence of hypertension or diabetes than patients without (Table 2). In univariate analysis, age, male sex, preoperative ischemic presentation, hypertension, and diabetes all showed significant association with postoperative hyperperfusion. The multivariate logistic regression analysis demonstrated that preoperative ischemic presentation (OR 5.73, 95\% CI $1.27-25.88, \mathrm{p}=0.023)$ and admission $\mathrm{mRS}$ score (OR $1.81,95 \%$ CI $1.06-3.10, \mathrm{p}=0.031$ ) were significantly associated with postoperative symptomatic CHS (Table 4).

\section{Postoperative Complications and Long-Term Clinical Outcomes}

After excluding 41 revascularizations $(6.7 \%)$ due to loss of the patients to follow-up and 37 revascularizations (6.2\%) with less than 1 month of available followup, 532 revascularizations were included in the analysis of outcomes. A total of 25 stroke events occurred during follow-up. We did not observe a significant difference in stroke-free survival between the indirect, direct, or combined bypass groups $(p=0.308)$. We then matched 67 revascularizations with postoperative complications to 67 
TABLE 1. Baseline characteristics of patients with and without postoperative complications

\begin{tabular}{|c|c|c|c|c|}
\hline \multirow[b]{2}{*}{ Characteristic } & \multirow[b]{2}{*}{ All Pts $(n=610)$} & \multicolumn{2}{|c|}{ Postop Complications } & \multirow[b]{2}{*}{$p$ Value } \\
\hline & & Present $(n=74)$ & Absent $(n=536)$ & \\
\hline Mean age at op (yrs) & $37.5 \pm 9.4$ & $39.6 \pm 9.7$ & $37.3 \pm 9.3$ & 0.047 \\
\hline \multicolumn{5}{|l|}{ Sex } \\
\hline Male & $267(43.8)$ & $42(56.8)$ & $255(42.0)$ & 0.016 \\
\hline Female & $343(56.2)$ & $32(43.2)$ & $311(58.0)$ & \\
\hline \multicolumn{5}{|l|}{ Onset symptoms } \\
\hline Ischemic & $371(60.8)$ & $62(83.8)$ & $309(57.6)$ & 0.001 \\
\hline Infarction & $211(34.6)$ & $37(50.0)$ & $174(32.5)$ & \\
\hline TIA & $160(26.2)$ & $25(33.8)$ & $135(25.2)$ & \\
\hline Hemorrhagic & $196(32.1)$ & $8(10.8)$ & $188(35.1)$ & \\
\hline Nonspecific & $43(7.0)$ & $4(5.4)$ & $39(7.3)$ & \\
\hline Headache & $28(4.6)$ & $2(2.7)$ & $26(4.9)$ & \\
\hline Epilepsy & $10(1.6)$ & $1(1.4)$ & $9(1.7)$ & \\
\hline Asymptomatic & $5(0.8)$ & $1(1.4)$ & $4(0.7)$ & \\
\hline \multicolumn{5}{|l|}{ Admission mRS score } \\
\hline Mean & $1.4 \pm 0.8$ & $1.5 \pm 0.8$ & $1.4 \pm 0.8$ & 0.389 \\
\hline $0-2$ & $557(91.3)$ & 66 (89.2) & $491(91.6)$ & 0.489 \\
\hline $3-6$ & $53(8.7)$ & $8(10.8)$ & $45(8.4)$ & \\
\hline \multicolumn{5}{|l|}{ Past medical history } \\
\hline Hypertension & $168(27.5)$ & $27(36.5)$ & $141(26.3)$ & 0.066 \\
\hline Diabetes & $39(6.4)$ & $10(13.5)$ & $29(5.4)$ & 0.018 \\
\hline \multicolumn{5}{|l|}{ Disease involved } \\
\hline Bilateral & $536(87.9)$ & $69(93.2)$ & $467(87.1)$ & 0.131 \\
\hline Unilateral & $74(12.1)$ & $5(6.8)$ & $69(12.9)$ & \\
\hline \multicolumn{5}{|l|}{ Suzuki stage*† } \\
\hline Mean & $3.8 \pm 1.0$ & $4.0 \pm 0.9$ & $3.7 \pm 1.0$ & 0.017 \\
\hline 1 & $0(0.0)$ & $0(0.0)$ & $0(0.0)$ & 0.03 \\
\hline 2 & $42(7.9)$ & $1(1.6)$ & $41(8.7)$ & \\
\hline 3 & $174(32.6)$ & $17(27.0)$ & $157(33.3)$ & \\
\hline 4 & $197(36.9)$ & $24(38.1)$ & $173(36.7)$ & \\
\hline 5 & $103(19.3)$ & $20(31.7)$ & $83(17.6)$ & \\
\hline 6 & $18(3.4)$ & $1(1.6)$ & $17(3.6)$ & \\
\hline Posterior circulation involvement* & $179(33.5)$ & $28(44.4)$ & $151(32.1)$ & 0.05 \\
\hline \multicolumn{5}{|l|}{ Surgery type } \\
\hline Indirect bypass & $199(32.6)$ & $25(33.8)$ & $174(32.5)$ & 0.726 \\
\hline Direct bypass & $291(47.7)$ & $37(50.0)$ & $254(47.4)$ & \\
\hline Combined & $120(19.7)$ & $12(16.2)$ & $108(20.1)$ & \\
\hline Mean LOS (days) & $19.6 \pm 6.2$ & $26.2 \pm 8.8$ & $18.7 \pm 5.1$ & $<0.0001$ \\
\hline \multicolumn{5}{|l|}{ Discharge mRS score } \\
\hline Mean & $1.2 \pm 0.8$ & $1.8 \pm 1.1$ & $1.1 \pm 0.8$ & $<0.0001$ \\
\hline $0-2$ & $564(92.5)$ & $56(75.7)$ & $508(94.8)$ & $<0.0001$ \\
\hline $3-6$ & $46(7.5)$ & $18(24.3)$ & $28(5.2)$ & \\
\hline
\end{tabular}

LOS = length of hospital stay; pts = patients.

Values are numbers of cases (\%) unless otherwise indicated. Mean values are presented with SDs.

* Preoperative DSA was performed in 433 patients (before 534 surgical procedures).

$\dagger$ In this and successive tables, Suzuki stage refers to the Suzuki stage of the surgically treated side.

revascularizations without postoperative complications according to propensity scores. The clinical characteristics and radiographic features of these 2 groups are presented in Table 5. The matched groups were compared to ensure that no significant differences were present between these 2 groups after propensity-score matching. In the 67 cases of revascularization with postoperative complications, 18 patients (24.3\%) had mRS scores $>2$ at 
TABLE 2. Characteristics of patients with and without postoperative ischemia and CHS

\begin{tabular}{|c|c|c|c|c|c|c|}
\hline \multirow[b]{2}{*}{ Characteristic } & \multicolumn{3}{|c|}{ Infarction } & \multicolumn{3}{|c|}{ Hyperperfusion } \\
\hline & Present $(n=30)$ & Absent $(n=580)$ & p Value & Present $(n=27)$ & Absent $(n=583)$ & $p$ Value \\
\hline Mean age at op (yrs) & $39.1 \pm 9.9$ & $37.5 \pm 9.3$ & 0.368 & $41.3 \pm 10.0$ & $37.4 \pm 9.3$ & 0.035 \\
\hline \multicolumn{7}{|l|}{ Sex } \\
\hline Female & $16(53.3)$ & $327(56.4)$ & 0.743 & $9(33.3)$ & $334(57.3)$ & 0.014 \\
\hline Male & $14(46.7)$ & $253(43.6)$ & & $18(66.7)$ & $249(42.7)$ & \\
\hline Ischemic presentation & $27(90.0)$ & $344(59.3)$ & 0.001 & $25(92.6)$ & $346(59.3)$ & 0.001 \\
\hline Admission mRS score & $1.6 \pm 09$ & $1.4 \pm 0.8$ & 0.208 & $1.7 \pm 0.8$ & $1.4 \pm 0.8$ & 0.098 \\
\hline \multicolumn{7}{|l|}{ Mean } \\
\hline $0-2$ & $26(86.7)$ & $531(91.6)$ & 0.319 & $23(85.2)$ & $534(91.6)$ & 0.281 \\
\hline $3-6$ & $4(13.3)$ & $49(8.4)$ & & $4(14.8)$ & $49(8.4)$ & \\
\hline \multicolumn{7}{|l|}{ Past medical history } \\
\hline Hypertension & $9(30.0)$ & $159(27.4)$ & 0.757 & $12(44.4)$ & $156(26.8)$ & 0.044 \\
\hline Diabetes & $4(13.3)$ & $35(6.0)$ & 0.117 & $6(22.2)$ & $33(5.7)$ & 0.005 \\
\hline \multicolumn{7}{|l|}{ Disease involvement } \\
\hline Unilateral & $2(6.7)$ & $72(12.4)$ & 0.564 & $2(7.4)$ & $72(12.3)$ & 0.761 \\
\hline Bilateral & $28(93.3)$ & $508(95.1)$ & & $25(92.6)$ & $511(87.7)$ & \\
\hline \multicolumn{7}{|l|}{ Suzuki stage* } \\
\hline Mean & $4.3 \pm 0.8$ & $3.8 \pm 1.0$ & 0.008 & $3.8 \pm 0.9$ & $3.8 \pm 1.0$ & 0.838 \\
\hline 1 & $0(0.0)$ & $0(0.0)$ & 0.005 & $0(0.0)$ & $0(0.0)$ & \\
\hline 2 & $0(0.0)$ & $42(8.3)$ & & $1(4.5)$ & $41(8.0)$ & \\
\hline 3 & $4(15.4)$ & $170(33.5)$ & & $7(31.8)$ & $167(32.6)$ & \\
\hline 4 & $12(46.2)$ & $185(36.4)$ & & $9(40.9)$ & $188(36.7)$ & \\
\hline 5 & $9(34.6)$ & $94(18.5)$ & & $5(22.7)$ & 98 (19.1) & \\
\hline 6 & $1(3.8)$ & $17(3.3)$ & & $0(0.0)$ & $18(3.5)$ & \\
\hline PCA involvement* & $13(50.0)$ & $166(32.7)$ & 0.068 & $9(40.9)$ & $170(33.2)$ & 0.453 \\
\hline \multicolumn{7}{|l|}{ Operation side } \\
\hline Left & $20(66.7)$ & $296(51.0)$ & 0.095 & & & \\
\hline Right & $10(33.3)$ & $284(49.0)$ & & & & \\
\hline \multicolumn{7}{|l|}{ Surgery type } \\
\hline Indirect bypass & $11(36.7)$ & $188(32.4)$ & 0.682 & $8(29.6)$ & $191(32.8)$ & 0.383 \\
\hline Direct bypass & $12(40.0)$ & $279(48.1)$ & & $16(59.3)$ & $275(47.2)$ & \\
\hline Combined bypass & $7(23.3)$ & $113(19.5)$ & & $3(11.1)$ & $117(20.1)$ & \\
\hline Mean LOS (days) & $26.03 \pm 9.8$ & $19.3 \pm 5.8$ & 0.001 & $24.1 \pm 5.9$ & $19.4 \pm 6.2$ & $<0.0001$ \\
\hline Mean discharge mRS score & $2.2 \pm 1.0$ & $1.1 \pm 1.0$ & $<0.0001$ & $1.7 \pm 1.0$ & $1.1 \pm 0.9$ & $<0.0001$ \\
\hline
\end{tabular}

discharge. Compared with patients without postoperative complications, patients who experienced any postoperative complication had longer hospital stays $(\mathrm{p}<0.0001)$ and worse mRS scores at discharge $(\mathrm{p}<0.0001)$. KaplanMeier analysis (by hemisphere, Fig. 1) showed no significant difference in stroke-free survival between the postoperative complications and no-complication groups $(\mathrm{p}=$ 0.513 ) during follow-up. The overall stroke rate was 2.13 per 100 patient-years for the complications group and 1.77 per 100 patient-years for the no-complications group. At the final follow-up (mean time since surgery $27.8 \pm 16.5$ months), 14 (77.8\%) of 18 patients with complications and mRS scores $>2$ at discharge had recovered to mRS score $<2$. Patients who had postoperative complications were more likely to have functional disability (follow-up mRS score $>2$ ) than those without postoperative complications $(11.9 \%$ vs $4.5 \%, \mathrm{p}=0.116)$. The overall $\mathrm{mRS}$ scores at admission, discharge, and last follow-up are shown in Fig. 2 for the complications group and no-complications groups.

\section{Discussion}

Our study demonstrates that postoperative complications occurred in $12.1 \%$ (74 of 610) of the revascularization procedures in adult patients with MMD. The most common postoperative complications were new ischemia and CHS. Advanced Suzuki stage was an independent risk factor for postoperative ischemia, and mRS score on admission was independently associated with postoperative CHS. In addition, preoperative ischemic presentation was 
TABLE 3. Logistic regression analysis for postoperative ischemia

\begin{tabular}{|c|c|c|c|c|c|c|c|c|}
\hline \multirow{3}{*}{$\begin{array}{l}\text { Characteristic } \\
\text { Age at op }\end{array}$} & \multicolumn{4}{|c|}{ Univariate Analyses } & \multicolumn{4}{|c|}{ Multivariate Analyses } \\
\hline & \multirow{2}{*}{$\frac{\mathrm{OR}}{1.02}$} & \multicolumn{2}{|c|}{$95 \% \mathrm{Cl}$} & \multirow{2}{*}{$\frac{p \text { Value }}{0.339}$} & \multirow{2}{*}{$\frac{\mathrm{OR}}{1.04}$} & \multicolumn{2}{|c|}{$95 \% \mathrm{Cl}$} & \multirow{2}{*}{$\frac{p \text { Value }}{0.165}$} \\
\hline & & 0.98 & 1.06 & & & 0.99 & 1.09 & \\
\hline Male sex & 1.13 & 0.54 & 2.36 & 0.743 & 1.07 & 0.46 & 2.47 & 0.883 \\
\hline Ischemic presentation & 6.17 & 1.85 & 20.59 & 0.003 & 5.85 & 1.65 & 20.65 & 0.006 \\
\hline Admission mRS score & 1.32 & 0.86 & 2.02 & 0.209 & 0.91 & 0.49 & 1.68 & 0.766 \\
\hline \multicolumn{9}{|l|}{ Past medical history } \\
\hline Hypertension & 1.14 & 0.51 & 2.53 & 0.757 & 0.57 & 0.21 & 1.57 & 0.274 \\
\hline Diabetes & 2.40 & 0.79 & 7.25 & 0.122 & 1.98 & 0.54 & 7.25 & 0.302 \\
\hline \multicolumn{9}{|l|}{ Disease involvement } \\
\hline Unilateral & Ref & Ref & Ref & & Ref & Ref & Ref & \\
\hline Bilateral & 1.98 & 0.46 & 8.51 & 0.356 & 2.45 & 0.31 & 19.45 & 0.395 \\
\hline Suzuki stage & 1.75 & 1.16 & 2.65 & 0.008 & 1.67 & 1.06 & 2.63 & 0.027 \\
\hline PCA involvement & 2.06 & 0.93 & 4.54 & 0.073 & 2.05 & 0.88 & 4.78 & 0.097 \\
\hline \multicolumn{9}{|l|}{ Surgery type } \\
\hline Indirect bypass & Ref & Ref & Ref & & Ref & Ref & Ref & \\
\hline Direct bypass & 0.74 & 0.32 & 1.70 & 0.472 & 0.75 & 0.28 & 2.01 & 0.564 \\
\hline Combined bypass & 1.06 & 0.40 & 2.81 & 0.909 & 1.26 & 0.44 & 3.67 & 0.668 \\
\hline
\end{tabular}

an independent predictor for both postoperative ischemia and CHS. Moreover, postoperative complications were significantly associated with worse outcomes at discharge, although this effect seem to be diminished in follow-up.

The cerebral vascular supply of MMD is characterized by a dynamic transitional state of conversion of the internal carotid system to the external carotid system (IC-EC conversion). ${ }^{11,40}$ Revascularization is employed for treatment of MMD to complement the "IC-EC conversion" and thus prevent secondary events. Surgical complications of
MMD include both neurological (postoperative cerebral infarction and CHS) and nonneurological complications. ${ }^{11}$ Cerebral ischemia shortly after surgery represents a serious postoperative complication. The previously reported rate of postoperative ischemia in adult MMD patients varies from $1.5 \%$ to $11.4 \%$ for direct/combined bypass ${ }^{12,18,20}$ and from $3.0 \%$ to $11.1 \%$ for indirect bypass. ${ }^{21,26,30} \mathrm{~A}$ recent meta-analysis showed that perioperative ischemia (cerebral ischemic event within 30 days of bypass) rates by procedure type were $4.8 \%$ for indirect bypass, $3.2 \%$ for direct

TABLE 4. Logistic regression analysis for postoperative CHS

\begin{tabular}{|c|c|c|c|c|c|c|c|c|}
\hline \multirow{3}{*}{$\begin{array}{l}\text { Characteristics } \\
\text { Age at op }\end{array}$} & \multicolumn{4}{|c|}{ Univariate } & \multicolumn{4}{|c|}{ Multivariate } \\
\hline & \multirow{2}{*}{$\frac{\mathrm{OR}}{1.05}$} & \multicolumn{2}{|c|}{$95 \% \mathrm{Cl}$} & \multirow{2}{*}{$\frac{p \text { Value }}{0.036}$} & \multirow{2}{*}{$\begin{array}{c}\mathrm{OR} \\
1.02\end{array}$} & \multicolumn{2}{|c|}{$95 \% \mathrm{Cl}$} & \multirow{2}{*}{$\frac{p \text { Value }}{0.523}$} \\
\hline & & 1.00 & 1.09 & & & 0.96 & 1.08 & \\
\hline Male sex & 2.68 & 1.19 & 6.07 & 0.018 & 2.51 & 0.95 & 6.62 & 0.063 \\
\hline Ischemic presentation & 8.56 & 2.01 & 36.49 & 0.004 & 5.73 & 1.27 & 25.88 & 0.023 \\
\hline Admission mRS score & 1.44 & 0.93 & 2.23 & 0.101 & 1.81 & 1.06 & 3.10 & 0.031 \\
\hline \multicolumn{9}{|l|}{ Past medical history } \\
\hline Hypertension & 2.19 & 1.00 & 4.78 & 0.049 & 1.30 & 0.48 & 3.51 & 0.606 \\
\hline Diabetes & 4.76 & 1.80 & 12.60 & 0.002 & 1.75 & 0.47 & 6.47 & 0.402 \\
\hline \multicolumn{9}{|l|}{ Disease involved } \\
\hline Unilateral & Ref & Ref & Ref & & Ref & Ref & Ref & \\
\hline Bilateral & 1.76 & 0.41 & 7.59 & 0.448 & 2.91 & 0.36 & 23.67 & 0.319 \\
\hline Suzuki stage & 1.05 & 0.67 & 1.63 & 0.838 & 1.04 & 0.65 & 1.67 & 0.872 \\
\hline PCA involvement & 1.39 & 0.58 & 3.32 & 0.455 & 1.11 & 0.44 & 2.81 & 0.826 \\
\hline \multicolumn{9}{|l|}{ Surgery type } \\
\hline Indirect bypass & Ref & Ref & Ref & & Ref & Ref & Ref & \\
\hline Direct bypass & 1.39 & 0.58 & 3.31 & 0.458 & 1.16 & 0.42 & 3.22 & 0.778 \\
\hline Combined bypass & 0.61 & 0.16 & 2.35 & 0.475 & 0.37 & 0.07 & 1.93 & 0.236 \\
\hline
\end{tabular}


TABLE 5. Comparison of patient characteristics in the complications and no-complications groups after propensityscore matching

\begin{tabular}{|c|c|c|c|c|}
\hline \multirow[b]{2}{*}{ Characteristics } & \multirow[b]{2}{*}{ All Pts } & \multicolumn{2}{|c|}{ Postop Complications } & \multirow[b]{2}{*}{$p$ Value } \\
\hline & & Present $(n=67)$ & Absent $(n=67)$ & \\
\hline Mean age at op (yrs) & $38.7 \pm 9.0$ & $38.9 \pm 9.4$ & $38.5 \pm 8.7$ & 0.797 \\
\hline \multicolumn{5}{|l|}{ Sex } \\
\hline Male & $75(56.0)$ & $39(58.2)$ & $36(53.7)$ & 0.602 \\
\hline Female & $59(44.0)$ & $28(41.8)$ & $31(46.3)$ & \\
\hline \multicolumn{5}{|l|}{ Onset symptoms } \\
\hline Ischemic & $109(81.3)$ & $56(83.6)$ & $53(79.1)$ & 0.801 \\
\hline Infarction & $67(50.0)$ & $32(47.8)$ & $35(52.2)$ & \\
\hline TIA & $42(31.3)$ & $24(35.8)$ & $18(26.9)$ & \\
\hline Hemorrhagic & $16(11.9)$ & $7(10.4)$ & $9(13.4)$ & \\
\hline Nonspecific & $9(6.7)$ & $4(6.0)$ & $5(7.5)$ & \\
\hline Headache & $7(5.2)$ & $2(3.0)$ & $5(7.5)$ & \\
\hline Epilepsy & $1(0.7)$ & $1(1.5)$ & $0(0.0)$ & \\
\hline Asymptomatic & $1(0.7)$ & $1(1.5)$ & $0(0.0)$ & \\
\hline \multicolumn{5}{|l|}{ Admission mRS score } \\
\hline Mean & $1.5 \pm 0.8$ & $1.5 \pm 0.8$ & $1.5 \pm 0.7$ & 0.661 \\
\hline $0-2$ & $121(90.3)$ & $59(88.1)$ & $62(92.5)$ & 0.381 \\
\hline $3-6$ & $13(9.7)$ & $8(11.9)$ & $5(7.5)$ & \\
\hline \multicolumn{5}{|l|}{ Past medical history } \\
\hline Hypertension & $46(34.3)$ & $23(34.3)$ & $23(34.3)$ & 1.000 \\
\hline \multicolumn{5}{|l|}{ Diabetes } \\
\hline \multicolumn{5}{|l|}{ Disease involvement } \\
\hline Bilateral & $123(91.8)$ & $62(92.5)$ & $61(91.0)$ & 0.753 \\
\hline Unilateral & $11(8.2)$ & $5(7.5)$ & $6(9.0)$ & \\
\hline \multicolumn{5}{|c|}{ Suzuki stage of surgery side* } \\
\hline Mean & $4.0 \pm 0.8$ & $4.0 \pm 0.8$ & $4.1 \pm 0.8$ & 0.567 \\
\hline 1 & $2(1.5)$ & $1(1.5)$ & $1(1.5)$ & 0.869 \\
\hline 2 & $25(18.7)$ & $14(20.9)$ & $11(16.4)$ & \\
\hline 3 & $21(15.7)$ & $11(16.4)$ & $10(14.9)$ & \\
\hline 4 & $50(37.3)$ & $23(34.3)$ & $27(40.3)$ & \\
\hline 5 & $32(23.9)$ & $17(25.4)$ & $15(22.4)$ & \\
\hline 6 & $4(3.0)$ & $1(1.5)$ & $3(4.5)$ & \\
\hline PCA involvement* & $47(35.1)$ & $26(38.8)$ & $21(31.3)$ & 0.570 \\
\hline \multicolumn{5}{|l|}{ Surgery type } \\
\hline Indirect bypass & $52(38.8)$ & $25(37.3)$ & $27(40.3)$ & 0.598 \\
\hline Direct bypass & $64(47.8)$ & $31(46.3)$ & $33(49.3)$ & \\
\hline Combined bypass & $18(13.4)$ & $11(16.4)$ & $7(10.4)$ & \\
\hline Mean LOS (days) & $22.3 \pm 7.6$ & $25.3 \pm 7.9$ & $19.3 \pm 6.0$ & $<0.0001$ \\
\hline \multicolumn{5}{|l|}{ Discharge mRS score } \\
\hline Mean & $1.5 \pm 1.0$ & $1.8 \pm 1.1$ & $1.1 \pm 0.8$ & $<0.0001$ \\
\hline $0-2$ & $113(84.3)$ & $49(73.1)$ & $64(95.5)$ & $<0.0001$ \\
\hline $3-6$ & $21(15.7)$ & $18(26.9)$ & $3(4.5)$ & \\
\hline
\end{tabular}

bypass, and $4.1 \%$ for combined bypass. ${ }^{39}$ The frequency of postoperative ischemia in our study $(5.5 \%$ for indirect bypass, $4.1 \%$ for direct bypass, and $5.8 \%$ for combined bypass) is consistent with rates reported in the previous reports, showing an almost equal distribution of ischemic events after surgery for different bypass types.

Attempts have been made to identify risk factors for postoperative ischemia. Frequent occurrence of preopera- 


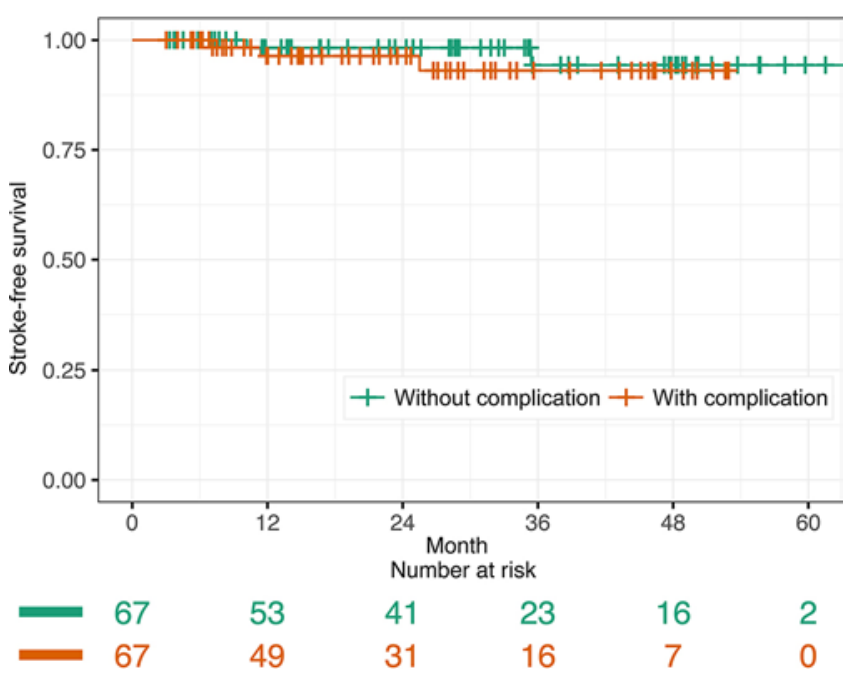

FIG. 1. Kaplan-Meier plot showing freedom from stroke per hemisphere treated for adult MMD patients with and without complications after propensity-score matching. Tick marks indicate time points after which data were censored for a particular patient-hemisphere in the group (point of last follow-up). There was no significant difference in freedom from stroke between the 2 groups ( $p=0.513$, log-rank test). Figure is available in color online only.

tive TIAs has also been suggested as an important indicator of perioperative ischemic complication risk. ${ }^{18}$ Other factors, such as female sex, ${ }^{22}$ hypotension, hypocapnia, perioperative hypovolemia, brain atrophy, ${ }^{25,37}$ presence of underlying disease, ${ }^{12}$ and disease involvement in the PCA,${ }^{20}$ have been previously reported to be associated with increase of postoperative ischemic complications in MMD patients. Controversy still remains in the literature regarding which contribute as risk factors. In our study, higher Suzuki stage and a preceding ischemic presentation were verified to be independent risk factors associated with new ischemia after revascularization, findings that are in line with previous reports. The relationship between these risk factors and postoperative ischemia is likely due to cerebral hemodynamic compromise. Patients with an advanced Suzuki stage and those with a preoperative ischemic presentation are more likely to have poor collateralization pathways to compensate for the hemodynamic impairment. Surgical procedures may induce stress on hemodynamic regulation and thus lead to ischemic complications. Besides the hemodynamic issue, postoperative development of new bypass flow could stimulate occlusion of the stenotic ICA or proximal MCA, leading to thrombotic occlusion. ${ }^{28}$ Thromboembolism from the anastomosed artery could also result in hypoperfusion at the associated peripheral vascular territory. ${ }^{8}$ In addition, swelling of the temporal muscle used for indirect bypass is another possible cause of cerebral ischemia during the acute stage.

Another common neurological complication after surgical treatment of MMD is CHS. Hyperperfusion syndrome in MMD was first reported by Uno et al. in a patient after extracranial-intracranial bypass in $1998 .{ }^{42}$ It has been reported that patients with MMD have a significantly higher risk for CHS after bypass surgery than patients with other atherosclerotic occlusive cerebrovascular diseases. ${ }^{10}$ Moreover, studies on postoperative CHS in MMD patients suggest that most CHS occurred in patients after direct bypass procedures in moyamoya disease. ${ }^{44}$ The incidence of symptomatic CHS in adult MMD patients has been reported at rates ranging from $6.7 \%$ to $38.2 \%$.6,10,24,33 The incidence of radiological hyperperfusion could be high as $67.5 \% .^{41}$ In contrast to previous studies, the incidence of symptomatic CHS in the present study is $4.0 \%$ for indirect bypass and $4.6 \%$ for direct/combined bypass. One possible reason for this relatively low rate of postoperative CHS is that we strictly maintained the systolic blood pressure of all patients at $120 \mathrm{~mm} \mathrm{Hg}$ to $130 \mathrm{~mm} \mathrm{Hg}$ in the perioperative period. Intravenous urapidil was administered intermittently or infused continuously in hypertensive patients, if necessary. Controlling the systolic blood pressure to between $110 \mathrm{~mm} \mathrm{Hg}$ and $130 \mathrm{~mm} \mathrm{Hg}$ was previously reported to reduce the risk of CHS to $6.7 \% .^{6}$ Our results provide further evidence of the importance of appropriate blood pressure management in the perioperative period. However, it should be noted that with the lowering of blood pressure, the risk of cerebral ischemia might increase. Maintaining the patient's postoperative blood pressure near their preoperative level may be recommended. ${ }^{20}$ The use of free-radical scavengers could be an additional treatment to counteract secondary brain injury due to CHS. ${ }^{32}$ Further studies would be necessary to determine the optimal perioperative blood pressure management. Interestingly, 8 patients were found to have CHS in our indirect bypass group. It was previously thought that 3-4 months were required for development of surgical collaterals after indirect bypass surgery. ${ }^{16}$ However, the studies on which that belief was based did not examine changes in cerebral blood flow shortly after surgery. In an experimental study, angiogenesis was reported to be established within 1 week after indirect revascularization. ${ }^{31} \mathrm{~A}$ recent study demonstrated that mean transit time delay began to decrease within 1 week after indirect bypass in MMD patients. ${ }^{17}$ Furthermore, Cho et al. ${ }^{3}$ reported that some moyamoya patients who presented with postoperative transient neurological deterioration after indirect surgery showed significantly increased cerebral blood flow in both the operated hemisphere and the focal hyperperfusion area compared with their preoperative findings. Some mechanisms were speculated: 1) release of growth factors or neuropeptides can cause a focal vasodilating effect and 2) increase in CBF may result from focal neuronal excitability by some factors, such as insulin-like growth factor and fibroblast growth factor. ${ }^{3}$ These findings raise interesting questions regarding the immediate postoperative period after indirect revascularization. At present, a unified definition has not been established for CHS in MMD patients who have undergone revascularization surgery. CHS is usually characterized by unilateral headache, face pain, seizures, and transient neurological deficits. ${ }^{43}$ The clinical signs of CHS in our series of MMD patients included fluctuating aphasia, dysarthria, and numbness/weakness in the limb contralateral to the anatomical site of the anastomosis. Although patients did not have preoperative seizures and antiepileptic drugs were administered postoperatively, we cannot completely exclude the possibility that some CHS 

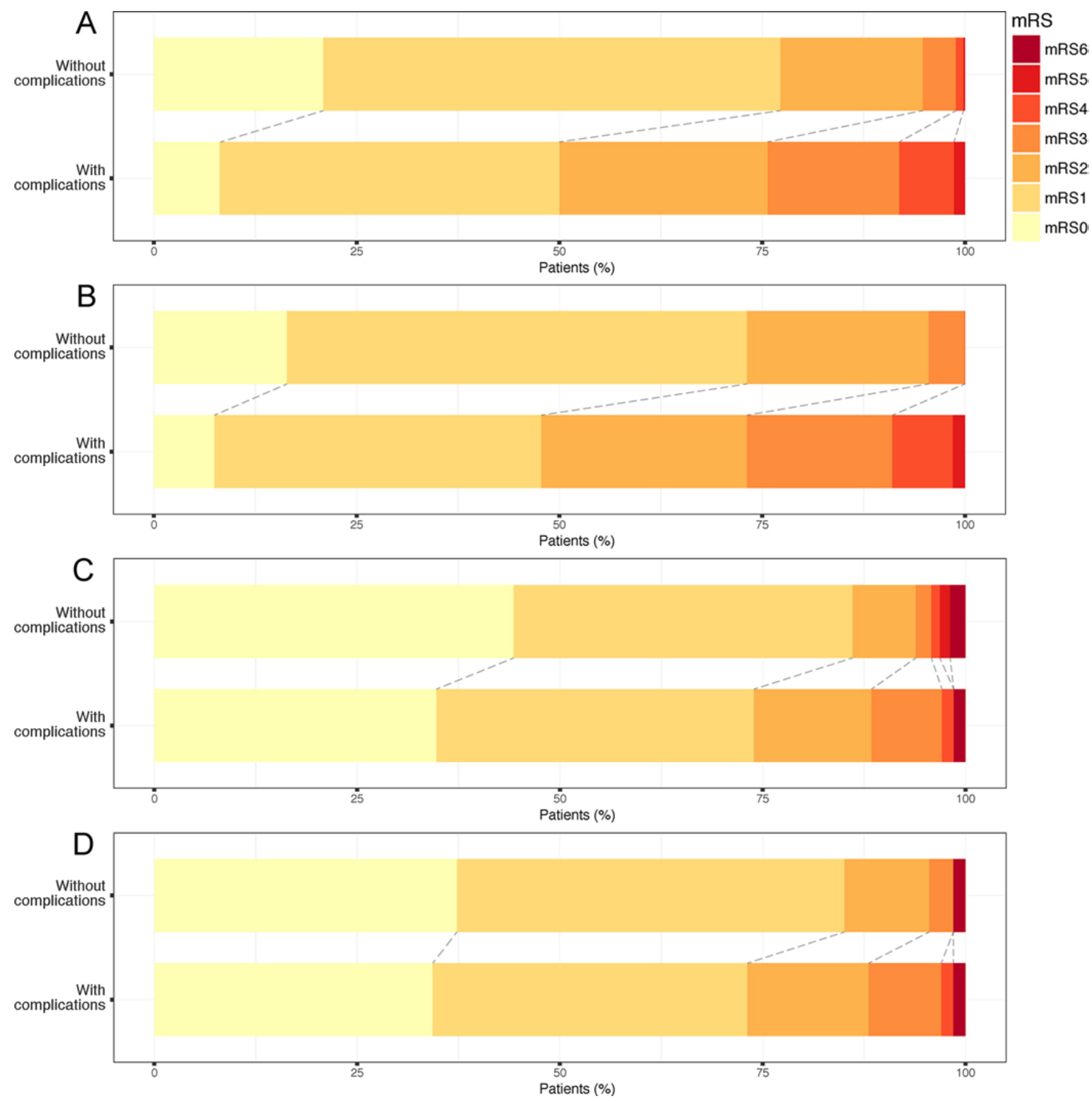

FIG. 2. Comparison of $m R S$ scores of patients with and without complications. The proportions of patients with $m R S$ scores ranging from 0 (yellow) to 6 (dark red) are shown for all patients at discharge (A), patients in the propensity-score matched groupsscores at discharge (B), all patients at last follow-up (C), and patients in the propensity-score matched groups-scores at last follow-up (D). Figure is available in color online only.

patients in the indirect bypass group could have had partial or complex seizures. Presumably, the neurological deficits in these patients with hyperperfusion without postoperative hemorrhage are transient. Those neurological deficits were usually observed within 1 week after the surgery and resolved before discharge in our study. However, a previous study found that asymptomatic or transient cerebral hyperperfusion can impair cognitive function. ${ }^{15}$ Future studies focusing on cognitive impairment in the perioperative period may provide more information to guide the management of surgically treated MMD patients.

Risk factors for postoperative CHS have been less well studied than those for postoperative ischemia. Preoperative ischemic presentation and higher admission $\mathrm{mRS}$ score were found to be independent risk factors for postoperative CHS in our study. This is in accordance with a 
previous study which concluded that symptomatic hyperperfusion occurred more often in ischemic-onset patients than in hemorrhagic-onset patients. ${ }^{14}$ On the contrary, Fujimura et al. ${ }^{9}$ stated that patients with adult-onset disease and those with hemorrhagic onset are at significantly higher risk for postoperative hyperperfusion. Differences in the patient populations, CHS detection methods, surgical techniques, and perioperative management may contribute to the discrepancy. Since patients with preoperative ischemic presentation are prone to both postoperative ischemia and hyperperfusion, the perioperative management of those patients should be treated with caution. It is important to identify CHS because the treatment for it is the opposite of that of ischemia. A patient with postoperative CHS would require a tighter control of blood pressure, whereas a patient with postoperative ischemia will most likely require an increase in blood pressure and perfusion. Clarification of the mechanisms of CHS and postoperative ischemia in MMD is imperative for development of optimal perioperative management to avoid postoperative complications, including both CHS and cerebral ischemia.

The difference in surgical efficacy between different bypass techniques remains controversial in the treatment of adult MMD. Direct or combined bypass procedures have been recommended in adult MMD patients because these surgeries seem to be more effective at preventing future stroke risk than indirect bypass in these patients. ${ }^{19,27}$ However, it has been postulated that direct bypass may result in higher rates of postoperative complications than indirect bypass. The direct bypass is more technically challenging, and operation takes more time. Although direct bypass can immediately increase cerebral blood flow in the chronically ischemic brain, it could also lead to hyperperfusion. In addition, direct bypass is more invasive and requires temporary vascular occlusion during the anastomosis, which can lead to postoperative ischemia. A pooled data analysis of 967 cases showed that the incidence of postoperative hemorrhage within 30 days after revascularization was significantly lower in patients treated with indirect bypass compared with those who underwent direct or combined bypasses..$^{39} \mathrm{~A}$ recent meta-analysis investigating surgical outcomes of symptomatic MMD patients showed that perioperative complications were more frequent in the direct bypass group $(30.2 \%)$ than in the indirect bypass group (18.8\%), but the difference did not reach statistical significance $(p=0.176){ }^{35}$ In our study, we did not see a significant difference in the overall postoperative complication rate when comparing the different surgical modalities.

The long-term prognosis of postoperative complications is unclear. We found that although postoperative complications may cause unfavorable short-term outcomes, they might not have a significant impact on longterm outcomes. Though there was a shift toward worse neurological status in the complication group compared with the group without postoperative complications, the difference did not reach statistical significance. This result may in part be due to insufficient statistical power in the long-term analysis or the lack of sensitivity of the mRS for more subtle deficits. Since most events are related to hemodynamic imbalance, complications in MMD are rarely disastrous. Reportedly, over 75\% of MMD patients have a benign course without significant daily dysfunction after surgical treatment. ${ }^{5}$ Although the benefit of direct bypass has been reported in treating patients with adult-onset MMD presenting with ischemia, ${ }^{27}$ no significant difference in stroke-free survival between the different surgical modalities was found in patients with various presentations in our group. Multicenter randomized controlled trials comparing different surgical or medical treatments are needed to elucidate outcomes for adult MMD patients. A notable finding in our study was that patients with postoperative hemorrhages in the CHS group were most likely to have recurrent strokes and worse outcomes, but there was not enough power to draw definitive conclusions due to the relatively small number of patients with postoperative hemorrhage.

Several potential limitations of our study should be noted. This is a single-center study, and selection bias existed. There is potential bias in patient selection for the different surgical procedures. To reduce the imbalance of baseline characteristics and maximize the power of the study, we conducted propensity-score matching for the follow-up analysis. Although many clinical factors were recorded in our data, hematocrit values and intraoperative measures were not analyzed in this study. Nevertheless, to the best of our knowledge, this is the largest study to investigate complications of revascularizations in MMD. Further studies are required to assess the optimal perioperative management to reduce postoperative complications.

\section{Conclusions}

Our study demonstrates that postoperative complications occurred after $12.1 \%$ of the revascularization procedures performed for treatment of adult patients with MMD. We found that advanced Suzuki stage and preoperative ischemic presentation were independent risk factors for postoperative ischemia and that admission mRS score and preoperative ischemic presentation were independently associated with postoperative CHS. Postoperative complications were significantly associated with worse outcomes at discharge. No significant differences in stroke-free survival and neurological status were detected between patients with and without postoperative complications during follow-up.

\section{Acknowledgments}

This study was supported by "13th Five-Year Plan" National Science and Technology Supporting Plan (2015BAI12B04), the National Natural Science Foundation of China (81371292), and the Beijing Municipal Administration of Hospitals' Mission Plan (SML20150501).

\section{References}

1. Antonucci MU, Burns TC, Pulling TM, Rosenberg J, Marks MP, Steinberg GK, et al: Acute preoperative infarcts and poor cerebrovascular reserve are independent risk factors for severe ischemic complications following direct extracranialintracranial bypass for moyamoya disease. AJNR Am J Neuroradiol 37:228-235, 2016

2. Cho WS, Kim JE, Kim CH, Ban SP, Kang HS, Son YJ, et al: Long-term outcomes after combined revascularization surgery in adult moyamoya disease. Stroke 45:3025-3031, 2014 
3. Cho WS, Lee HY, Kang HS, Kim JE, Bang JS, Oh CW: Symptomatic cerebral hyperperfusion on SPECT after indirect revascularization surgery for moyamoya disease. Clin Nucl Med 38:44-46, 2013

4. Deng X, Gao F, Zhang D, Zhang Y, Wang R, Wang S, et al: Effects of different surgical modalities on the clinical outcome of patients with moyamoya disease: a prospective cohort study. J Neurosurg [epub ahead of print July 7, 2017. DOI: 10.3171/2016.12.JNS162626], 2017

5. Fujimura M, Bang OY, Kim JS: Moyamoya disease. Front Neurol Neurosci 40:204-220, 2016

6. Fujimura M, Inoue T, Shimizu H, Saito A, Mugikura S, Tominaga T: Efficacy of prophylactic blood pressure lowering according to a standardized postoperative management protocol to prevent symptomatic cerebral hyperperfusion after direct revascularization surgery for moyamoya disease. Cerebrovasc Dis 33:436-445, 2012

7. Fujimura M, Kaneta T, Shimizu H, Tominaga T: Cerebral ischemia owing to compression of the brain by swollen temporal muscle used for encephalo-myo-synangiosis in moyamoya disease. Neurosurg Rev 32:245-249, discussion 249, 2009

8. Fujimura M, Kaneta T, Tominaga T: Efficacy of superficial temporal artery-middle cerebral artery anastomosis with routine postoperative cerebral blood flow measurement during the acute stage in childhood moyamoya disease. Childs Nerv Syst 24:827-832, 2008

9. Fujimura M, Mugikura S, Kaneta T, Shimizu H, Tominaga T: Incidence and risk factors for symptomatic cerebral hyperperfusion after superficial temporal artery-middle cerebral artery anastomosis in patients with moyamoya disease. Surg Neurol 71:442-447, 2009

10. Fujimura M, Shimizu H, Inoue T, Mugikura S, Saito A, Tominaga T: Significance of focal cerebral hyperperfusion as a cause of transient neurologic deterioration after extracranialintracranial bypass for moyamoya disease: comparative study with non-moyamoya patients using N-isopropyl-p-[ $\left.{ }^{123} \mathrm{I}\right]$ iodoamphetamine single-photon emission computed tomography. Neurosurgery 68:957-965, 2011

11. Fujimura M, Tominaga T: Current status of revascularization surgery for moyamoya disease: special consideration for its 'internal carotid-external carotid (IC-EC) conversion' as the physiological reorganization system. Tohoku J Exp Med 236: $45-53,2015$

12. Guzman R, Lee M, Achrol A, Bell-Stephens T, Kelly M, Do HM, et al: Clinical outcome after 450 revascularization procedures for moyamoya disease. Clinical article. J Neurosurg 111:927-935, 2009

13. Hayashi K, Horie N, Suyama K, Nagata I: Incidence and clinical features of symptomatic cerebral hyperperfusion syndrome after vascular reconstruction. World Neurosurg 78:447-454, 2012

14. Hayashi T, Shirane R, Fujimura M, Tominaga T: Postoperative neurological deterioration in pediatric moyamoya disease: watershed shift and hyperperfusion. J Neurosurg Pediatr 6:73-81, 2010

15. Hirooka R, Ogasawara K, Sasaki M, Yamadate K, Kobayashi M, Suga Y, et al: Magnetic resonance imaging in patients with cerebral hyperperfusion and cognitive impairment after carotid endarterectomy. J Neurosurg 108:1178-1183, 2008

16. Houkin K, Nakayama N, Kuroda S, Ishikawa T, Nonaka T: How does angiogenesis develop in pediatric moyamoya disease after surgery? A prospective study with MR angiography. Childs Nerv Syst 20:734-741, 2004

17. Ishii Y, Tanaka Y, Momose T, Yamashina M, Sato A, Wakabayashi S, et al: Chronological evaluation of cerebral hemodynamics by dynamic susceptibility contrast magnetic resonance imaging after indirect bypass surgery for moyamoya disease. World Neurosurg 108:427-435, 2017

18. Iwama T, Hashimoto N, Tsukahara T, Murai B: Peri-opera- tive complications in adult moyamoya disease. Acta Neurochir (Wien) 132:26-31, 1995

19. Jeon JP, Kim JE, Cho WS, Bang JS, Son YJ, Oh CW: Metaanalysis of the surgical outcomes of symptomatic moyamoya disease in adults. J Neurosurg 128:793-799, 2018

20. Jung YJ, Ahn JS, Kwon DH, Kwun BD: Ischemic complications occurring in the contralateral hemisphere after surgical treatment of adults with moyamoya disease. J Korean Neurosurg Soc 50:492-496, 2011

21. Kazumata K, Ito M, Tokairin K, Ito Y, Houkin K, Nakayama $\mathrm{N}$, et al: The frequency of postoperative stroke in moyamoya disease following combined revascularization: a single-university series and systematic review. J Neurosurg 121:432440, 2014

22. Khan N, Achrol AS, Guzman R, Burns TC, Dodd R, BellStephens T, et al: Sex differences in clinical presentation and treatment outcomes in moyamoya disease. Neurosurgery 71:587-593, 2012

23. Kim JE, Jeon JS: An update on the diagnosis and treatment of adult Moyamoya disease taking into consideration controversial issues. Neurol Res 36:407-416, 2014

24. Kim JE, Oh CW, Kwon OK, Park SQ, Kim SE, Kim YK: Transient hyperperfusion after superficial temporal artery/ middle cerebral artery bypass surgery as a possible cause of postoperative transient neurological deterioration. Cerebrovasc Dis 25:580-586, 2008

25. Kim SH, Choi JU, Yang KH, Kim TG, Kim DS: Risk factors for postoperative ischemic complications in patients with moyamoya disease. J Neurosurg 103 (5 Suppl):433-438, 2005

26. Kim T, Oh CW, Bang JS, Kim JE, Cho WS: Moyamoya disease: treatment and outcomes. J Stroke 18:21-30, 2016

27. Kim T, Oh CW, Kwon OK, Hwang G, Kim JE, Kang HS, et al: Stroke prevention by direct revascularization for patients with adult-onset moyamoya disease presenting with ischemia. J Neurosurg 124:1788-1793, 2016

28. Kuroda S, Houkin K, Nunomura M, Abe H: Frontal lobe infarction due to hemodynamic change after surgical revascularization in moyamoya disease-two case reports. Neurol Med Chir (Tokyo) 40:315-320, 2000

29. Liu X, Zhang D, Shuo W, Zhao Y, Wang R, Zhao J: Long term outcome after conservative and surgical treatment of haemorrhagic moyamoya disease. J Neurol Neurosurg Psychiatry 84:258-265, 2013

30. Mallory GW, Bower RS, Nwojo ME, Taussky P, Wetjen NM, Varzoni TC, et al: Surgical outcomes and predictors of stroke in a North American white and African American moyamoya population. Neurosurgery 73:984-992, 2013

31. Nakamura M, Imai H, Konno K, Kubota C, Seki K, Puentes $\mathrm{S}$, et al: Experimental investigation of encephalomyosynangiosis using gyrencephalic brain of the miniature pig: histopathological evaluation of dynamic reconstruction of vessels for functional anastomosis. Laboratory investigation. J Neurosurg Pediatr 3:488-495, 2009

32. Ogasawara K, Inoue T, Kobayashi M, Endo H, Fukuda T, Ogawa A: Pretreatment with the free radical scavenger edaravone prevents cerebral hyperperfusion after carotid endarterectomy. Neurosurgery 55:1060-1067, 2004

33. Ohue S, Kumon Y, Kohno K, Watanabe H, Iwata S, Ohnishi $\mathrm{T}$ : Postoperative temporary neurological deficits in adults with moyamoya disease. Surg Neurol 69:281-287, 2008

34. Pandey P, Steinberg GK: Neurosurgical advances in the treatment of moyamoya disease. Stroke 42:3304-3310, 2011

35. Qian C, Yu X, Li J, Chen J, Wang L, Chen G: The efficacy of surgical treatment for the secondary prevention of stroke in symptomatic moyamoya disease: a meta-analysis. Medicine (Baltimore) 94:e2218, 2015

36. Research Committee on the Pathology and Treatment of Spontaneous Occlusion of the Circle of Willis: Guidelines for 
diagnosis and treatment of moyamoya disease (spontaneous occlusion of the circle of Willis). Neurol Med Chir (Tokyo) 52:245-266, 2012

37. Sato K, Shirane R, Yoshimoto T: Perioperative factors related to the development of ischemic complications in patients with moyamoya disease. Childs Nerv Syst 13:68-72, 1997

38. Scott RM, Smith ER: Moyamoya disease and moyamoya syndrome. N Engl J Med 360:1226-1237, 2009

39. Sun H, Wilson C, Ozpinar A, Safavi-Abbasi S, Zhao Y, Nakaji P, et al: Perioperative complications and long-term outcomes after bypasses in adults with moyamoya disease: a systematic review and meta-analysis. World Neurosurg 92:179-188, 2016

40. Suzuki J, Takaku A: Cerebrovascular "moyamoya" disease. Disease showing abnormal net-like vessels in base of brain. Arch Neurol 20:288-299, 1969

41. Uchino H, Kuroda S, Hirata K, Shiga T, Houkin K, Tamaki $\mathrm{N}$ : Predictors and clinical features of postoperative hyperperfusion after surgical revascularization for moyamoya disease: a serial single photon emission CT/positron emission tomography study. Stroke 43:2610-2616, 2012

42. Uno M, Nakajima N, Nishi K, Shinno K, Nagahiro S: Hyperperfusion syndrome after extracranial-intracranial bypass in a patient with moyamoya disease-case report. Neurol Med Chir (Tokyo) 38:420-424, 1998

43. van Mook WN, Rennenberg RJ, Schurink GW, van Oostenbrugge RJ, Mess WH, Hofman PA, et al: Cerebral hyperperfusion syndrome. Lancet Neurol 4:877-888, 2005
44. Zhao WG, Luo Q, Jia JB, Yu JL: Cerebral hyperperfusion syndrome after revascularization surgery in patients with moyamoya disease. Br J Neurosurg 27:321-325, 2013

\section{Disclosures}

The authors report no conflict of interest concerning the materials or methods used in this study or the findings specified in this paper.

\section{Author Contributions}

Conception and design: J Zhao, S Wang. Acquisition of data: M Zhao. Analysis and interpretation of data: M Zhao, Deng, S Wang. Drafting the article: M Zhao. Critically revising the article: Y Zhang. Reviewed submitted version of manuscript: J Zhao, D Zhang, R Wang. Approved the final version of the manuscript on behalf of all authors: J Zhao. Statistical analysis: R Wang. Administrative/technical/material support: R Wang. Study supervision: J Zhao.

\section{Correspondence}

Jizong Zhao: Beijing Tiantan Hospital, Capital Medical University, Beijing, China. zhaojz205@163.com. 